\title{
MASKING EFFECT OF PEPPERMINT FRAGRANCE STIMULUS ON CAR HORN SOUND STIMULUS WHILE DRIVING IN A GRAPHIC DRIVING SIMULATOR
}

\author{
Pil Kee Min ${ }^{1}$, Tsutomu Fujinami ${ }^{1}$, Hyung-Sik Kim ${ }^{2}$, Jun Bin $\mathrm{Ko}^{3}$ \\ and Cheol Kee Min ${ }^{4 *}$ \\ ${ }^{1}$ Human Life Design, Graduate School of Advanced Science and Technology, \\ Japan Advanced Institute of Science and Technology (JAIST), Nomi, \\ Ishikawa, Japan \\ ${ }^{2}$ Department of Biomedical Engineering, College of Science and Technology, \\ Konkuk University, Chungju, Chungbuk, Korea \\ ${ }^{3}$ Industry-University Cooperation Foundation, Hanbat National Univ., Korea \\ ${ }^{4}$ Department of Industrial \& Management Engineering, \\ Hanbat National Univ., Korea \\ ${ }^{1}$ pkmin88@gmail.com, ${ }^{1}$ fuji@jaist.ac.jp, ${ }^{2}$ hskim98@kku.ac.kr, \\ ${ }^{3}$ kjb1002@hanbat.ac.kr, ${ }^{*}$ mbc1473@ hanmail.net,
}

\begin{abstract}
In this study, autonomic nervous system responses including heart rate variability $(H R V)$ and galvanic skin response (GSR) were observed to examine the masking effect of peppermint fragrance stimulus on car horn sound stimulus in a graphic driving simulator. When the pre-experiment and post-experiment measurements for GSR were compared in car horn sound stimulus experiment (1) $(p<0.01)$, experiment (2) $(p<0.05)$, and experiment (3) $(p<0.01)$, driving experiment (2) $(p<0.01)$ and experiment $(3)(p<0.01)$, and peppermint fragrance stimulus experiment (3) $(p<0.05)$, the measurements of all the experiments increased with significant differences after the experiment. This suggests that when the car horn sound stimulus was presented, the sympathetic nervous system was activated and aroused. With respect to the $L F / H F$ ratio of the $H R V$, when a car horn sound stimulus was presented during driving, it showed different values depending on the presentation of peppermint fragrance. When the car horn sound stimulus was compared between before and after presenting peppermint fragrance while driving, it decreased with a significant difference $(p<0.05)$. In conclusion, the arousal level by the car horn sound stimulus while driving decreased due to the peppermint fragrance stimulus. Therefore, the peppermint fragrance stimulus is considered to have a masking effect on the car horn sound stimulus.
\end{abstract}

Keywords_- Graphic Driving Simulator, Peppermint, Car Horn Sound

\section{INTRODUCTION}

Car driving causes high level conscious concentration or tension for external stimuli, and as a result, the driver feels fatigue. Driving is a continuous series of complex behaviors that require various abilities including perception, decision making, and motor

Received: January 10, 2019

Reviewed: March 25, 2019

Accepted: April 1, 2019

${ }^{*}$ Corresponding Author 
functions. Furthermore, driving for a long time, intraday fluctuations of arousal level, lack of sleep, heat, noise, and vibrations from the car, toxic air in the car, tedious driving situation have been reported as the causes of driving fatigue [1].

In large cities, the adverse effects of road traffic noises on the entire city are more serious than other living noises due to the large number of vehicles and the extensive road networks [2]. As the traffic is becoming more complex, the frequency of using car horns as a means of communication among cars is gradually increasing. These car horn sounds are perceived as more unpleasant to the nearby pedestrians and drivers because they occur unexpectedly $[3,4]$.

Awareness about noise pollution, which is called "invisible pollution," has been low in the past compared to other types of pollutions, but awareness about noise pollution in modern cities is increasing significantly as it is becoming more serious [5]. Noises cause emotional anxiety such as "psychologically unstable" or "angry" [6], and also cause negative effects such as decreased task performance and low task satisfaction because they cause overload in cognitive processes [7, 8]. Physiologically, noise stimulates the sympathetic nerves of the autonomic nervous system and causes tension, thus leading to increased blood pressure, increased secretion of acidity, increased heart rate, and contraction of blood vessels [9]. Stress by noise causes various diseases including depression, anxiety, heart disease, and immune weakness [10, 11, 12]. In particular, mental illnesses by stress such as depression and anxiety suggest that noise is closely related to the mental health of modern people who live under the influence of various noises in modern society [13].

One method of reducing stress by external noises is to use the masking effect. In his book, Thomas [14] defined masking as "the obscuring of one sound by another". It can be understood that when we are exposed to two or more sounds, one of them is not heard well due to the other sounds. Masking can be explained best by the explanation that threshold of hearing of the smaller sound is increased by the larger sound. To review studies on masking effect, Loewen et al., [15] analyzed the effects of masked and unmasked office noises on arousal, stress, and cognitive performance. They found that performance was better in both simple and complex tasks in the case of masking than the other case, and that when the noise was masked, the subjects felt greater arousal, but they received less environmental disturbance and stress. Furthermore, the masking effect appeared when the background sound with high comfort was higher than the road traffic noise [16], and the water sound was effective in masking major noise sources in urban public spaces [17].

The above-mentioned studies demonstrated the masking effect of auditory stimuli with high comfort on the stress caused by noise.

The physiological reactions of the human body to noises can be measured by heart rate variability (HRV), electrooculogram (EOG), galvanic skin response (GSR), and photoplethysmogram (PPG), which are used as the objective indicators showing quantitative changes in the emotional state of people. Among them, the LF/HF (Low Frequency/High Frequency) ratio of the HRV quantifies the overall equilibrium between the parasympathetic nerve and sympathetic nerve, and represents the immediate reaction to external environment, and the GSR is used as an indicator of the change in the sympathetic nerve in response to stimuli [18, 19, 20, 21, 22].

Recently, many experimental evidences demonstrating that refreshing fragrances can affect the physiological reactions to task performance have been reported. A static feeling caused by a refreshing fragrance can help a person respond to negative reactions to stress [23]. Furthermore, attempts are being made to reduce stress caused by tasks using refreshing fragrances. The condition of spraying oxygen and fragrance during driving tended to be more psychologically comfortable, less tiring, and less burdensome physiologically than the control condition [24]. 
Subjects who were exposed to the fragrances, which are considered to be refreshing by fragrance judges or research participants, often maintain their arousal state and increase boundary task performance [25]. Moreover, refreshing fragrances promote remembrance of good memories [26], and fragrances affect the conflict and avoidance behaviors in interpersonal relationships [27]. Another study reported that the relaxed sense of the body caused by boring low-speed driving and the tension of the body caused by high-speed driving were reduced by fragrance stimuli [28].

Peppermint, which is used in aromatherapy, can be used as a steam inhalation method for digestive or respiratory diseases such as anorexia, vomiting, motion sickness, food poisoning, colitis, and diarrhea. Furthermore, peppermint has antiseptic, antibacterial, tonic, anti-inflammatory, antiseptic, and antioxidant effects; thus, it decreases fatigue, clears and refreshes the mind [29, 30]. Previous studies researched the masking effects of auditory stimuli on noise, but the present study examines the masking effect as one of other types of stimuli, that is, olfactory stimuli. In this study, the masking effects of the peppermint fragrance stimulus on the sense of tension due to external car horn sound stimulus while driving in a graphic driving simulator that has similar environment as the actual driving environment are examined by measuring the LF/HF ratio of the HRV and the GSR signals, and its effect on the reaction of autonomic nervous system is examined.

\section{EXPERIMENT METHOD}

\subsection{SUBJECTS}

The subjects were 11 male college students in their 20s with one year or longer driving experience. Their average age, height, and weight were $26.45 \pm 1.04$ years, $176.36 \pm 4.05 \mathrm{~cm}$, and $72.72 \pm 1.04 \mathrm{~kg}$, respectively. They had no problem in operating the graphic driving simulator, few simulator sickness symptoms, no smell and hearing disorders, and no disease related to autonomic nervous system. The subjects were prohibited anything that can affect the autonomic nervous system including drinking alcohol, smoking, and ingesting foods containing caffeine from 24 hours before the experiment.

\subsection{EXPERIMENTAL ENVIRONMENT AND EQUIPMENT}

The laboratory environment was maintained at an internal temperature of $24 \pm 2.1^{\circ} \mathrm{C}$ and a humidity of $25 \sim 45 \%$. The sound stimulus (ambulance siren, 70 75db, $30 \mathrm{sec}$ ) was presented by a speaker connected to a personal computer (HP Workstation xw4400), and Gom Player was used for the software for playing the sound. The physiological signals were measured using Biopac System's Biopac MP100 and Acqknowledge 3.8.1 was used for the analysis software.

\subsection{DRIVING SIMULATOR}

The driving simulator (GSD-300s Gridspace Co. Korea) installed inside the laboratory was used in this experiment and was configured to display the front, left, and right environments through three 32 -inch monitors as required for driving. The car model was "Click" from H Company with the same driving devices (steering wheel, accelerator pedal, brake pedal, parking brake, direction indicator lever, emergency light, wiper lever, headlight lever, gear lever, and safety belt) and display devices (direction indicators, speedometer, RPM meter, temperature gauge, fuel gauge, various warning lights, etc.,) were identical to the actual car. For the steering wheel, the motor driven power steering (MDPS) was used. The driving environment consisted of clear view, fine weather, and a three-lane one-way expressway <Fig. 1>. 


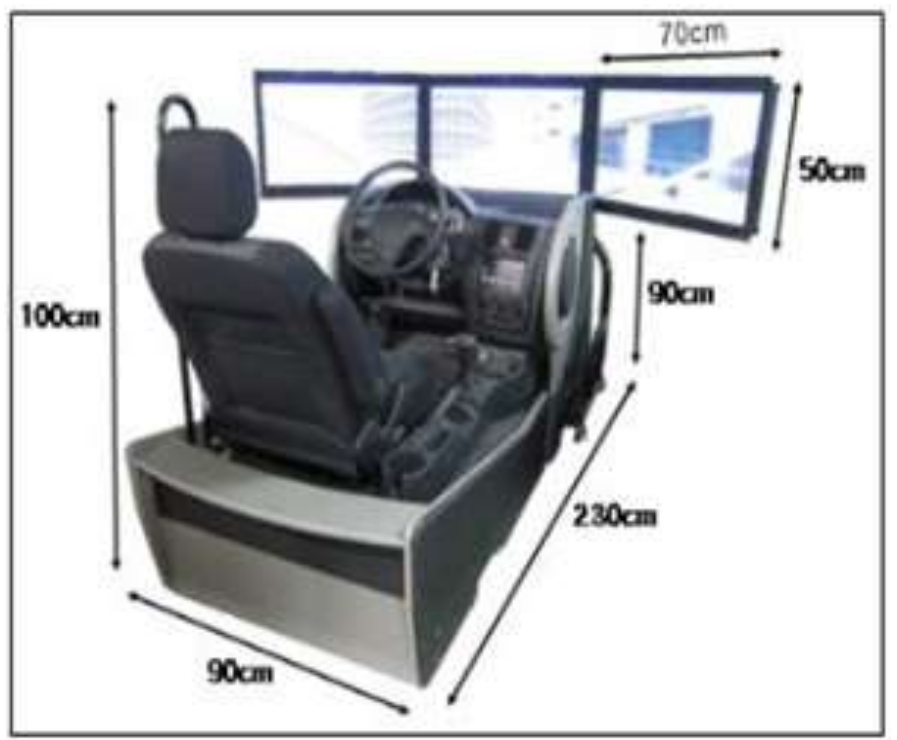

Fig. 1 Graphic Driving Simulator

\subsection{AUDITORY STIMULUS}

The auditory stimulus used in this experiment was the horn sound of an ambulance which was edited by the Movie Maker to last for 30 seconds. The volume of the sound stimulus was presented by adjusting the speaker volume to $70 \sim 75 \mathrm{db}$ with a noise meter (Os-11Onsoku Electric Inc).

\subsection{OLFACTORY STIMULUS}

Peppermint fragrance was used in this experiment. The subject in driving position smelled the fragrance from a closed container with the fragrance at $5 \mathrm{~cm}$ from the nose of the subject. To prevent contamination of the laboratory by the fragrance, the experiment was carried out after sufficient ventilation.

\subsection{DATA MEASUREMENT}

The physiological signals were measured using Biopac System MP100 and Acknowledge 3.8.1 after setting the sampling rate of $500 \mathrm{~Hz}$. The leads for ECG (electrocardiogram) were attached using the CM5 inducing method where the reference lead was attached to the right chest which is symmetrical to the + lead, and the + and leads were attached to the left chest and top of the breastbone, respectively <Figure 2>. The leads for GSR were attached to the second nodes of the left hand index and middle fingers. 


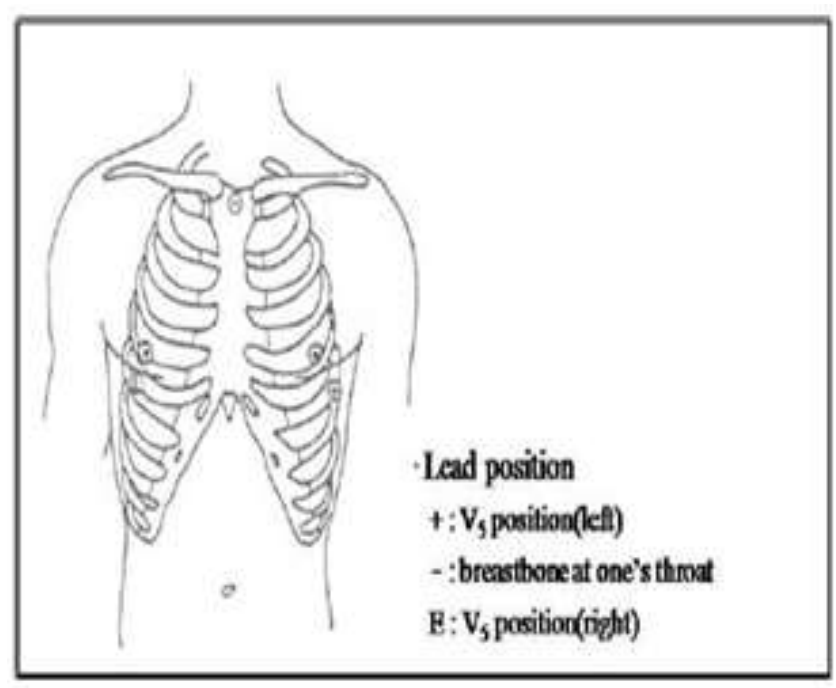

Fig. 2 CM5 Inducing Method

\subsection{EXPERIMENT PROCEDURE}

The experiment for this study was designed to investigate the masking effect of peppermint fragrance stimulus of the olfactory sense on the arousal state caused by car horn sound stimulus while driving in a graphic driving simulator through autonomic nervous system responses by measuring the LF/HF ratio of HRV and the GSR signals.

Before the experiment, anthropometric data were collected and the subjective body condition was checked on the day of experiment. Before starting the experiment, the subjects learned about the general contents of the experiment and conducted performance practice driving for about three minutes to adapt to the graphic driving simulator. Then they maintained stable condition after attaching the leads for measuring physiological signals. Figure 3 shows the experiment procedure. In Experiment (1), the physiological signals were measured while giving a sound stimulus for $30 \mathrm{sec}$ in stable condition. After taking a $10 \mathrm{~min}$ rest, in Experiment (2), the subject drove the car after $3 \mathrm{~min}$ of stable condition, and after $5 \mathrm{~min}$, a sound stimulus was presented for $30 \mathrm{sec}$. In Experiment (3), a fragrance stimulus was presented after $3 \mathrm{~min}$ of stable condition. Driving was performed after $3 \mathrm{~min}$, and a sound stimulus was presented after $5 \mathrm{~min}$. The driving speed was maintained at $75-85 \mathrm{~km}$.

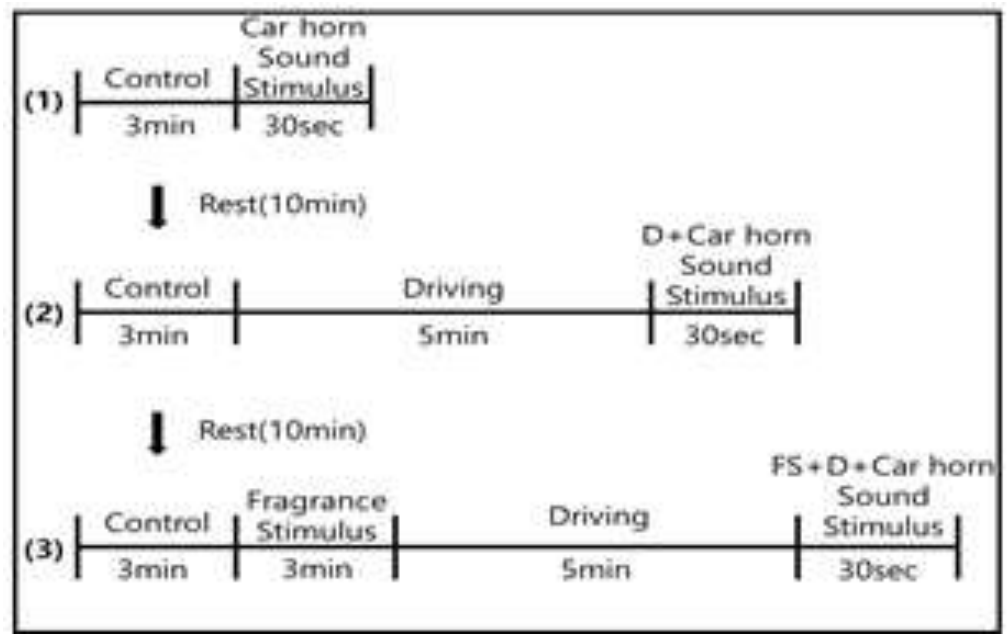

Fig. 3 Experimental Procedure 


\subsection{ANALYSIS METHOD}

ECG data were collected and analyzed with Acknowledge 3.8.1 by separating the signals before and after sound stimulus, driving, and fragrance stimulus for $30 \mathrm{sec}$ each. For frequency analysis, LF (0.04 0.15) and HF (0.15 0.4) were defined. The mean and standard deviation of LF/HF, which represents the ratio of activity of the sympathetic nerve system to the parasympathetic nervous system, were determined using Chart 5 . The GSR data were collected before and after sound stimulus, driving, and fragrance stimulus for $15 \mathrm{sec}$ each and the amplitude and standard deviation were determined. To observe the changes in the physiological signals caused by driving, sound stimulus, and fragrance stimulus, normalization was performed.

Normalization:

$$
(\%)=\frac{\text { Afterstimulus }- \text { Before stimulus }}{\text { Before stimulus }} \times 100
$$

For statistical analysis, the significance of the data collected before and after driving, sound stimulus, and fragrance stimulus was verified through t-test using SPSS ver. 12.0.

\section{RESULTS}

\subsection{GSR}

3.1.1. AUDITORY STIMULUS: In Experiment (1), when the car horn sound stimulus was presented in stable state, the GSR of post sound stimulus $(0.13 \pm 0.011)$ increased statistically significantly $(p<0.01)$ compared to pre sound stimulus $(0.0)$. In Experiment (2), when the car horn sound stimulus was presented during driving, the GSR of post sound stimulus $(0.11 \pm 0.17)$ increased statistically significantly $(p<0.05)$ compared to pre sound stimulus (0.00). In Experiment (3), when peppermint fragrance and car horn sound stimulus were presented during driving, the GSR of post sound stimulus $(0.06 \pm 0.04)$ increased statistically significantly $(\mathrm{p}<0.01)$ compared to pre sound stimulus (0.00). When the GSR values of post sound stimulus in Experiments (1) to (3) were compared, the difference between Experiments (1) and (3) was statistically insignificant, but the GSR in Experiment (3) tended to be lower <Fig. 4>.

3.1.2. DRIVING: In Experiment (2), with no fragrance condition, the GSR of post driving $(0.39 \pm 0.23)$ increased statistically significantly $(\mathrm{p}<0.01)$ compared to pre driving (0.00). In Experiment (3), in peppermint fragrance condition, the GSR of post driving $(0.33 \pm 0.22)$ increased statistically significantly $(\mathrm{p}<0.01)$ compared to pre driving $(0.00)$. The GSR values of post driving in Experiment (2) and (3) did not show a significant difference $\langle$ Fig. 5>.

3.1.3. OLFACTORY STIMULUS: In Experiment (3), the GSR of post fragrance stimulus $(0.11 \pm 0.13)$ increased statistically significantly $(\mathrm{p}<0.05)$ compared to the GSR of pre fragrance stimulus $(0.00)<$ Fig. $6>$. 


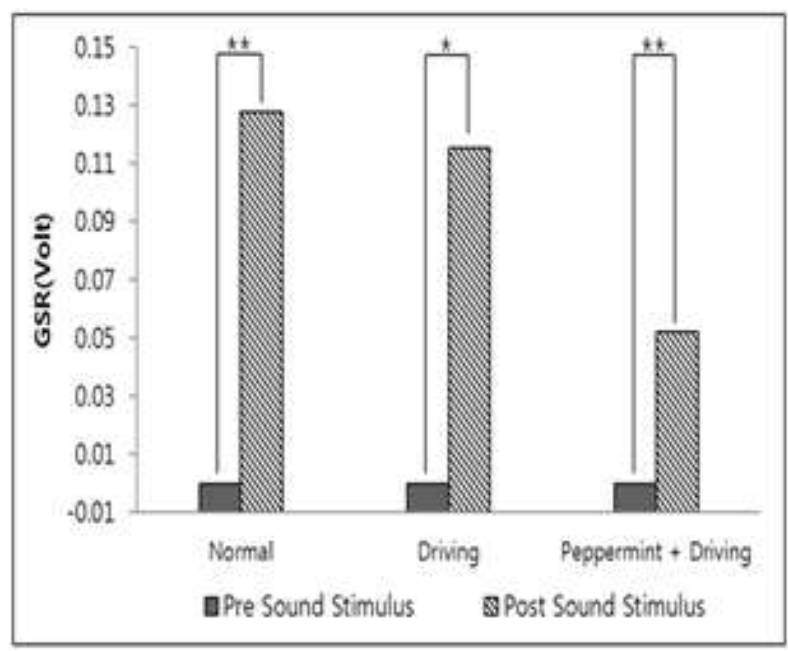

Fig. 4 Comparison of Pre \& Post Sound Stimulus (GSR) $\left(* * p<0.01,{ }^{*} p<0.05\right)$

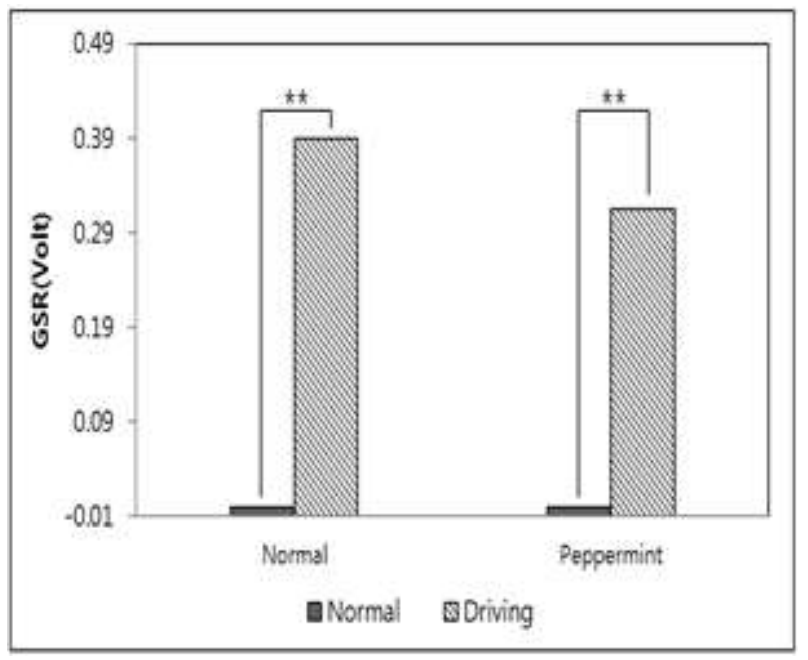

Fig. 5 Comparison of before and after presenting fragrance stimulus while Driving $(\mathrm{GSR})(* * \mathrm{p}<0.01)$

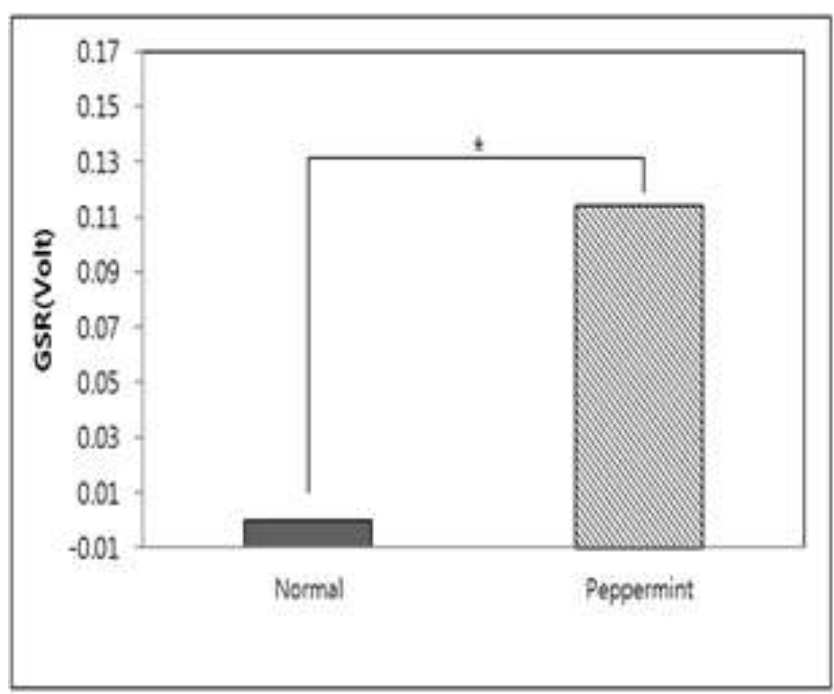

Fig. 6 Comparison of Pre \& Post Peppermint Fragrance Stimulus (GSR) $(* \mathrm{p}<0.05)$ 


\subsection{HRV}

3.2.1. AUDITORY STIMULUS: In Experiment (1), when car horn sound stimulus was presented in stable state, there was no statistically significant differences in HRV between pre sound stimulus $(0.0)$ and post sound stimulus $(4.35 \pm 6.97)$, but the HRV tended to be higher after presentation of the sound stimulus $(\mathrm{p}=0.068)$. In Experiment (2), when the car horn sound stimulus was presented during driving, the HRV of post sound stimulus $(2.48 \pm 3.26)$ increased statistically significantly $(\mathrm{p}<0.05)$ compared to pre sound stimulus (0.00). In Experiment (3), when car horn sound stimulus was presented during driving, the difference in HRV between pre sound stimulus $(0.00)$ and post sound stimulus $(0.02 \pm 0.95)$ was statistically insignificant. When the HRV values of Experiments (1) to (3) were compared, Experiments (1) and (3) showed no significant differences, but the value in Experiment (3) tended to be lower, and between Experiments (2) and (3), the value of Experiment (3) was statistically significantly lower $(\mathrm{p}<0.05)$ <Fig. 7>.

3.2.2. EXECUTION OF DRIVING: In Experiment (2), with no fragrance condition, the HRV of post driving $(11.78 \pm 17.75)$ was statistically significantly higher than the HRV of predriving (0.00) $(\mathrm{p}<0.05)$. In Experiment (3), with peppermint fragrance condition, the difference in HRV of pre driving $(0.00)$ and post driving $(9.08 \pm 15.17)$ was insignificant, but the HRV tended to increase. When Experiments (2) and (3) were compared, the HRVs of post driving did not show a statistically significant difference <Fig. 8>.

3.2.3. OLFACTORY STIMULUS: In Experiment (3), the HRVs before presenting the peppermint fragrance stimulus $(0.00)$ and after presenting the stimulus $(1.32 \pm 4.91)$ did not show a statistically significant difference <Fig. 9>.

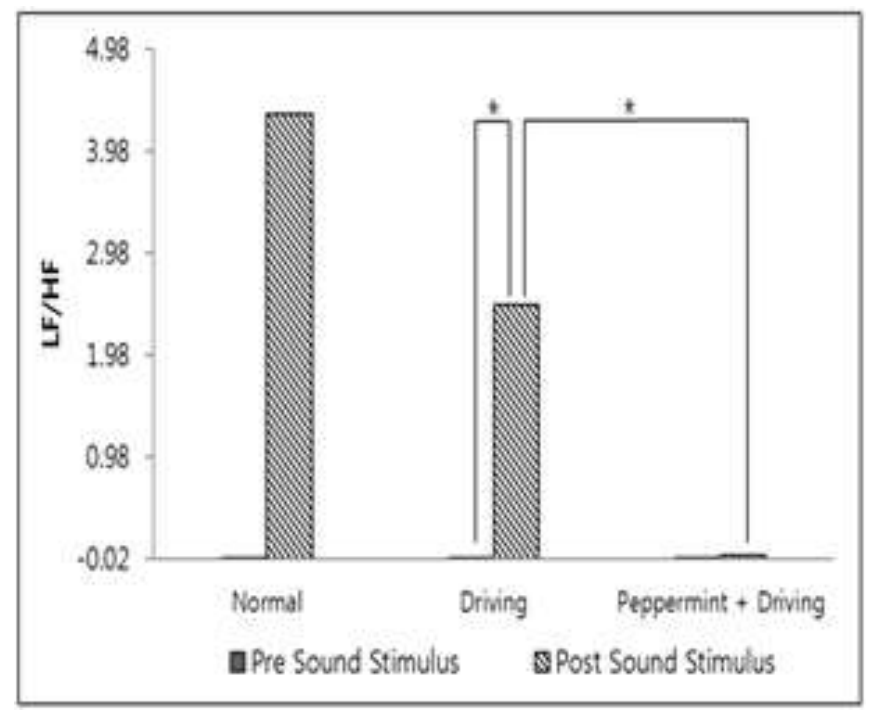

Fig. 7 Comparison of Pre \& Post Sound Stimulus (LF/HF) $\left({ }^{*} \mathrm{p}<0.05\right)$ 


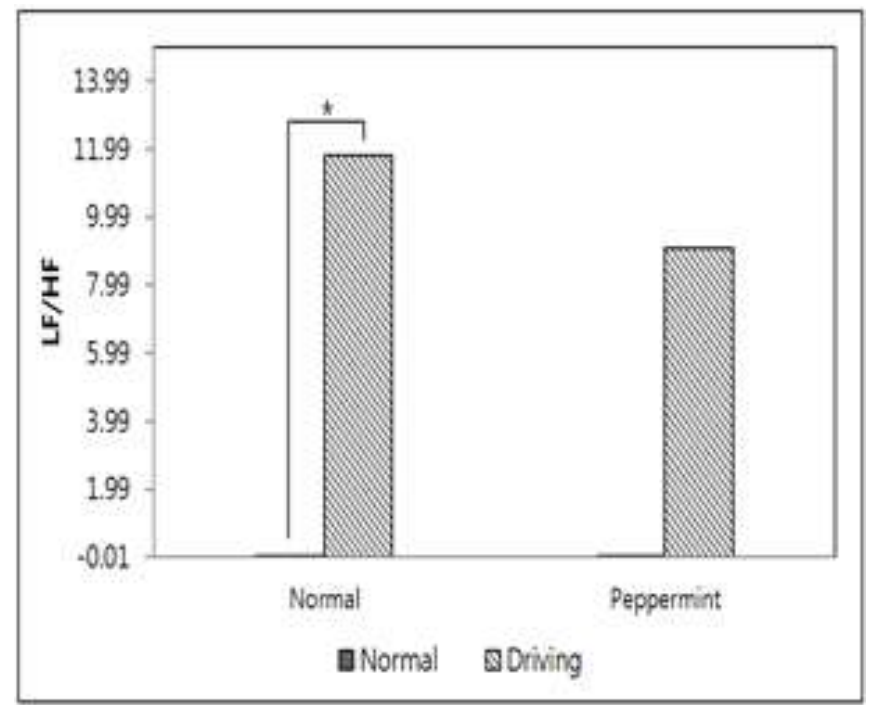

Fig. 8 Comparison of Before and After Presenting Fragrance Stimulus while Driving $(\mathrm{LF} / \mathrm{HF})(* \mathrm{p}<0.05)$

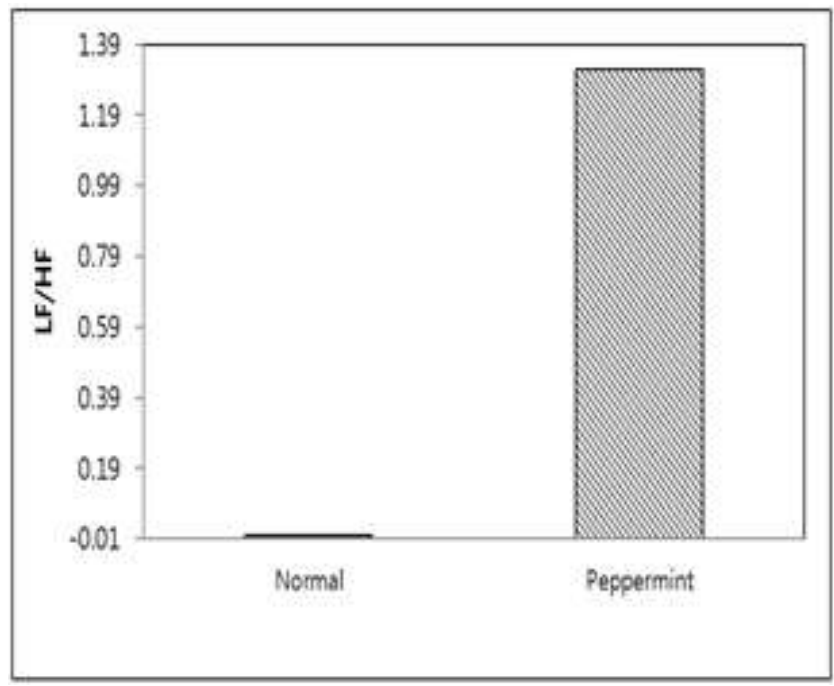

Fig. 9 Comparison of Pre \& Post Peppermint Fragrance Stimulus (LF/HF)

\section{CONCLUSIONS AND DISCUSSION}

As various machines, tools and automations have increased with industrial development, our living environments are exposed to more noises. Noise has begun to be noted as a principal culprit of pollution threatening human society [31]. People are receiving stress in complex social relationships and their health can be endangered by poor environments that are always exposed to severe noises [32].

Consequently, many studies are being conducted to reduce noises in living environment. Emotional criteria for various living noises are set [33], and noises are decreased by masking effect using sound [34]. Masking is a phenomenon in which the threshold of noise is changed by surrounding noises [35]. In other words, other sounds are not heard by a certain sound. Previous studies decreased noise by masking effect using sound, but in this study, the masking effect of an olfactory stimulus using a fragrance on car horn sound noise was examined.

The arousal state of drivers by car horn sound stimulus during driving was observed through changes in the autonomous nervous system. Furthermore, the effects of an 
olfactory stimulus using peppermint fragrance on the arousal state caused by a sudden car horn sound stimulus during driving were observed through GSR and HRV.

When GSR values were compared between before and after sound stimulus, driving, and fragrance stimulus, it increased statistically significantly after each stimulus. This means that the sympathetic nervous system was activated and the driver entered arousal state. However, peppermint fragrance did not have an effect on the arousal state caused by car horn sound stimulus during driving.

It is estimated that the above results may have been induced from the following situations.

First, the recovery time of tense activities acting on the entire nervous system takes several minutes, whereas the recovery time of the phasic activity takes a few seconds, which is short [36]. Therefore, adaptation to the fragrance stimulus appears to have affected the GSR signals. Second, individuals have different preferences on fragrances depending on the characteristics of individuals and fragrances [37, 38, 39]. Third, the psychological and physiological effects of simulator sickness can act as a significant distortion factor in emotional stimulus and evaluation research using a simulator [40].

The LF/HF ratio of HRV showed differences depending on the presentation of peppermint fragrance after a car horn sound stimulus was presented during driving. The LF/HF ratio decreased statistically significantly after peppermint fragrance was presented with a car horn sound stimulus during driving $(p<0.05)$. In other words, the activity of the sympathetic nervous system decreased, and the parasympathetic nervous system was activated. It can be considered that the arousal level caused by the car horn sound stimulus during driving was decreased by the peppermint fragrance. Therefore, the fragrance stimulus of the olfactory sense appears to have a masking effect on the noise. It can be concluded that the olfactory stimulus using a peppermint fragrance has a positive masking effect on the arousal state caused by car horn sound stimulus during driving.

However, in future studies, the preferences of subjects to fragrances need to be considered. Even refreshing fragrances may have different reactions depending on the individual preferences of the subjects. The reactions of the autonomous nerve system to fragrances are not uniform and may vary depending on the body condition and psychological state of each individual, and also by individual preferences or tastes for fragrances. Thus, these variables need to be considered sufficiently. Furthermore, a study is necessary to clearly determine whether the peppermint fragrance is an arousing or calming fragrance. The effects of lavender, which is known as a calming fragrance, and jasmine, which is known as an arousing fragrance, also need to be researched. The masking effects of olfactory stimulus using fragrances should be researched by adjusting the noise level, and the changes in the central nervous system as well as the autonomous nervous system need to be observed in order to observe the masking effects according to the noise level. Lastly, it is importance to obtain objective statistical data by increasing the number of subjects.

\section{REFERENCES}

[1] Sung, Eun Jung. "Influence of oxygen rate on driver fatigue during simulated driving." Korean Soc Emo Sens 5.1 (2002): 71-78.

[2] Lee, Nae-Hyun, Young-Min Park, and Young Sunwoo. "A Study on the Improvement of the Road Traffic Noise Prediction for Environmental Impact Assessment." Journal of Environmental Impact Assessment 10.4 (2001): 297-304.

[3] D.S.Ko, H.Kim, "Survey and Improvement of car Klaxon." Mokwon University Papers 21.4 (1992): 161-174.

[4] S.C.Lee, "Analysis of the relationship between horn-honking and driver's communication." Road Traffic Authority 10 (1991): 19-27.

[5] Ahn, Deug Soo. "Analyses on the cognitive effects of masking traffic noise by sounds of water." J Korean Inst Forest Recreat 6.4 (2002): 9-19.

[6] S.P.Kwon. "Environmental Science". Hyungseul Publishing 1985, pp.227, 272-278. 
[7] Broadbent, D. E. Human Performance and Noise, in C. M. Harris(ed.), Handbook of noise control, McGraw-Hill 1979.

[8] Poulton, E. Christopher. "Composite model for human performance in continuous noise." Psychological Review 86.4 (1979): 361-375.

[9] D.H.Sohn, "Effect of Noise in Human Body". Dongascience 1991; 67, pp. 103-107.

[10] Obelenis, Vytautas, and Vilija Malinauskienè. "The influence of occupational environment and professional factors on the risk of cardiovascular disease." Medicina 43.2 (2007): 96-102.

[11] Samad, Noreen, and Darakhshan Jabeen Haleem. "Serotonin-1A receptor responsiveness in stress and following adaptation to stress." Pakistan journal of pharmaceutical sciences 20.2 (2007): 115-119.

[12] Zheng, Kui-Cheng, and Makoto Ariizumi. "Modulations of immune functions and oxidative status induced by noise stress." Journal of occupational health 49.1 (2007): 32-38.

[13] W.K.Choi, K.S.Lee, H.Y.Joung, Y.C.Lee, J.H.Sohn, B.H.Lee, K.H.Pyun, I.S.Shim, "Low Frequency Noise Induces Stress Responses in the Rat" Korean Journal of the science of Emotion \& sensibility 10.3 (2007): 411-418

[14] Thomas D. Rossing. The Science of Sound. Addison-Wesley Publish Company 1990.

[15] Loewen, Laura J., and Peter Suedfeld. "Cognitive and arousal effects of masking office noise." Environment and Behavior 24.3 (1992): 381-395.

[16] H.Shin, S.K.Park, C.Kook, G.S.Jang. "The Masking Effects of Introducing Sounds about Road Traffic Noise." The Korean Society for Noise and Vibration Engineering Spring Conference, Korea, Spring 2005, pp. 599-602.

[17] J.Y.Heo, J.You, J.Y.Jeon. "Investigation of masking effect of water sounds on road traffic noise using semantic differential method." The Korean Society for Noise and Vibration Engineering Autumn Conference, Korea Autumn 2010, pp. 548-549.

[18] D.Y.Kim. "Development of human Sensibility Elements; Development of Human Auditory and Visual Sensory Measurement Technology and Construction of its Database." Ministry of Science and Technology (1998)

[19] H.J.Jeon, B.C.Min, E.J.Sung, C.J.Kim. "Evaluations on Driver's Sensibility Changes by Sudden Start and Sudden Stop Conditions in Driving Simulator." Korean Journal of the science of Emotion \& sensibility 5.4 (2002): 51-57.

[20] H.M.Sung, D.I.Cha, S.W.Kim, S.J.Park, C.J.Kim, Y.R.Yoon. "The study of driving fatigue using HRV analysis." Editors. Journal of Biomedical Engineering Research 24.1 (2003): 1-8.

[21] H.Y.Jang, J.H.Jang, T.S.Kim, C.S.Han, J.S.Han, J.Y.Ahn. "A Study on Driver's Physiological Response in Train Simulator." Journal of the Ergonomics Society of Korea 25.4 (2006): 129-135.

[22] M.C.Whang, J.E.Kim, C.J.kim. "Research about the Physiological signal changes in the auditory sensibility.” Ergonomics Society of Korea Spring Conference, Daegu, Korea, 18-19 April 1996. 1996, pp. 259-263.

[23] Y.H.Yoon, S.H.Kim, H.J.Lee, J.H.Lee, H.T.Kim. "The effects of task stressor, noise stimulus, conifer needle odor and soundguard on SCR, PPG and behavioral performance." Korean Journal of Clinical Psychology 16.2 (1997): 435-445.

[24] E.J.Sung, B.C.Min, H.J.Jeon, J.H.Jeon, Y.W.Nam, I.H.Kang, S.C.Kim, Y.K.Shin, C.J.Kim, "Effects of oxygen and aroma supply on driver fatigue." Ergonomics Society of Korea Conference, Yongpyong, Korea, Papers, November 2002, pp. 209-212.

[25] Warm, Joel S., William N. Dember, and R. Parasuraman. "Effects of olfactory stimulation on performance and stress." Journal of the Society Cosmetic Chemists 42.3 (1991): 199-210.

[26] Ehrlichman, Howard, and Jack N. Halpern. "Affect and memory: Effects of pleasant and unpleasant odors on retrieval of happy and unhappy memories." Journal of personality and social psychology 55.5 (1988): 769-779.

[27] Baron, Robert A. "Environmentally Induced Positive Affect: Its Impact on Self-Efficacy" Journal of Applied Social Psychology 20.4 (1990): 368-384.

[28] B.C.Min, Y.N.Kim, S.C.Chung, S.J.Kim, B.U.Min, C.J.Kim, M.K.Sin. "Autonomic Responses to Odorant Stimulation during Slow and Fast Vehicular Driving in Graphic Simulator." Korean Journal of the science of Emotion \& sensibility 3.1 (2000): 7-16.

[29] Ryoo, Jong-Won, and Bae-Cheon Cha. "Mineral content and antioxidative activity in some herb plants." Korean Journal of Medicinal Crop Science 6.1 (1998): 28-32.

[30] Y.N.Kim. "The study on effect of anti-stress managed by aroma essence oil therapy through olfactory." Master's Degree, Graduate School of alternative medicine, Kyongi University (2011).

[31] N.E.Bae, I.A.Choi. "The Effect of the Noise Level on the Stress Hormones during Aerobic Exercise.' Editors. Journal of Sport and Leisure Studies 34.2 (2008): 905-914.

[32] S.S.Kim, Y.S.Hong. "The Stress Hormones Responses Following Music Sound Levels during Aerobic Dances." Editors. Journal of Sport and Leisure Studies 12.1 (1999): 665-675.

[33] J.Y.Jeon, M.W.Koo, M.J.Cho. "A Study on Perceptual Evaluation of Noise Sources in Living Environment." The Korean Society for Noise and Vibration Engineering 11.3 (2001): 443-448.

[34] C.I.Jeong, J.You, P.J.Lee, J.Y.Jeon. "Effects of sound-masking on the soundscape of urban public spaces with multiple noises." The Korean Society for Noise and Vibration Engineering Spring Conference, Korea, Spring 2008, pp.258-261. 
[35] Pollack, Irwin. "Auditory informational masking." The Journal of the Acoustical Society of America 57.S5 (1975): 1-5

[36] S.D.Heo. "Characteristics of Auditory Evoked Electrodermal Activities.", Doctor of Medicine Degree, Graduate School of Dept. of Medicine, Dong-A University 2004.

[37] Ayabe-Kanamura S, Schicker I, Laska M, Hudson R, Distel H, Kobayakawa T and Saito S. "Differences in perception of everyday odors: a Japanese-German cross-cultural study." Chemical senses 23.1 (1998): 31-38.

[38] I.H.Kang, B.C.Min, K.J.Jeon, C.J.Kim. "Evaluation of the Odor with Aging." Editors. Korean Journal of the science of Emotion \& sensibility 5.2 (2002): 1-9.

[39] Stevens, Joseph C., and Natasha A. Spencer. "Olfactory detection of a complex versus a simple substance in advanced age." Chemical senses 19.4 (1994): 365-369.

[40] B.C.Min, S.C.Chung, E.J.Sung, H.J.Jeon, C.J.Kim. "Effects of Physiological Changes Evoked by Simulator Sickness on Sensibility Evaluation." Editors. Korean Journal of the science of Emotion \& sensibility 4.1 (2001): 23-31. 